\title{
The critical role of spin rotation in the giant magnetostriction of $\mathrm{La}(\mathrm{Fe}, \mathrm{Al})_{13}$
}

\author{
Yuzhu Song ${ }^{1 \dagger}$, Rongjin Huang ${ }^{2 \dagger}$, Ji Zhang ${ }^{3}$, Shantao Zhang ${ }^{3}$, Qingzhen Huang ${ }^{4}$, Shouguo Wang ${ }^{5}$, \\ Yong Jiang ${ }^{5}$ Laifeng $\mathrm{Li}^{2^{*}}$, Xianran Xing ${ }^{1}$ and Jun Chen ${ }^{1^{*}}$
}

\begin{abstract}
As an efficient converter between electromagnetic and mechanical energies, magnetostriction is an intriguing property for not only fundamental studies but also technological applications. However, the understanding of its microscopic origin remains challenging, which is critical for the development of magnetostriction materials. Here, the critical role of spin rotation in the giant magnetostriction of $\mathrm{La}(\mathrm{Fe}, \mathrm{Al})_{13}$ is first revealed by the in-situ magnetic and temperature field of neutron powder diffraction. The giant magnetostriction originates from magnetic-field-driven spin moment rotation of canting structure, in which the sharp increase of ferromagnetic component causes the elongation of icosahedron inside of lattice. Furthermore, it is the first time to reveal the accurate canting antiferromagnetic structure in $\mathrm{La}(\mathrm{Fe}, \mathrm{Al})_{13}$. The present study provides a new strategy, i.e., the spin rotation, for exploring new magnetostriction functional materials.
\end{abstract}

Keywords: magnetostriction, magnetic-field-induced spin rotation, magnetic structure, neutron powder diffraction

\section{INTRODUCTION}

Magnetostriction, a strain induced by magnetic field, is one of the frontier topics of advanced functional materials, to which much attention has been paid due to its unique role in various applications [1-11]. Magnetostrictive materials have been used as converters of electromagnetic energy into mechanical energy, such as actuators, motors, positioning devices, and energy har- vesting $[12,13]$. In order to meet the actual requirement, more applicable magnetostrictive materials need to be developed. After the discovery of the magnetostrictive effect in iron by Joule in 1842 [14], a great progress in the discovery of new magnetostriction materials has been made, such as rare-earth metals, Laves phase alloys $[15,16]$, Fe-Ga alloys [10,17-19], and Ni-Mn-Ga alloys [20,21].

Magnetostriction is a very complex phenomenon [1]. For example, the tetragonal distortion of the matrix induced by nanoheterogeneities causes large magnetostriction in Fe-Ga alloys [10,17-19], the magnetic-fieldcontrolled twin boundary motion induces large strain in $\mathrm{Ni}-\mathrm{Mn}-\mathrm{Ga}$ alloy [20,21], and the magnetic domain rotation results in giant magnetostriction in Laves phase alloys [15,16]. The phenomenological theory of magnetostriction can explain the change of ferromagnetic morphology from the perspective of macro deformation, but it cannot explain the intrinsic physical mechanism of magnetostriction [22]. It is demanded to study the magnetostriction from the microscopic level, such as crystal structure, bond length and atomic magnetic moment, so as to guide the exploration of magnetostriction materials. However, the origin of magnetostriction remains a mystery, which also hinders the design of new magnetostriction materials.

It is well known that magnetostriction is the change of lattice caused by the variation of magnetic field. Actually, due to the strong coupling between spin and lattice de-

\footnotetext{
${ }^{1}$ Beijing Advanced Innovation Center for Materials Genome Engineering, School of Mathematics and Physics, University of Science and Technology Beijing, Beijing 100083, China

${ }^{2}$ Key Laboratory of Cryogenics, Technical Institute of Physics and Chemistry, Chinese Academy of Sciences, Beijing 100190, China

${ }^{3}$ National Laboratory of Solid State Microstructures and Department of Materials Science and Engineering, College of Engineering and Applied Science \& Collaborative Innovation Center of Advanced Microstructures, Nanjing University, Nanjing 210093, China

${ }^{4}$ NIST Center for Neutron Research, National Institute of Standards and Technology, Gaithersburg MD, 20899-6102, USA

${ }^{5}$ School of Materials Science and Engineering, University of Science and Technology Beijing, Beijing 100083, China

${ }^{\dagger}$ These authors contributed equally to this work.

* Corresponding authors (emails: junchen@ustb.edu.cn (Chen J); 1fli@mail.ipc.ac.cn (Li L))
} 
grees of freedom, the modulation of lattice with the application of external field is generally influenced by such coupling. Thus, to better understand the mechanism of magnetostriction, the best way is to start with revealing spin lattice coupling, especially studying the response between lattice and spin under the external field.

Here, the origin of the giant magnetostriction property of $\mathrm{La}\left(\mathrm{Fe}_{11.5} \mathrm{Al}_{1.5}\right)$ is first revealed by the in-situ magnetic and temperature field of neutron powder diffraction (NPD) and macroscopic magnetic measurements. The magnetostriction of $\mathrm{La}\left(\mathrm{Fe}_{11.5} \mathrm{Al}_{1.5}\right)$ originates from the magnetic-field-driven spin moment rotation of canting structure. Interestingly, there is a strong correlation between lattice and the angle of spin moment. Furthermore, it is the first time to reveal the canting antiferromagnetic $(\mathrm{AFM})$ structure in $\mathrm{La}(\mathrm{Fe}, \mathrm{Al})_{13}$.

\section{EXPERIMENTAL SECTION}

The samples of single phase $\mathrm{La}\left(\mathrm{Fe}_{11.5} \mathrm{Al}_{1.5}\right)$ and $\mathrm{La}\left(\mathrm{Fe}_{11.3} \mathrm{Al}_{1.7}\right)$ were prepared by arc melting under a high purity argon atmosphere. After melting, the samples were annealed in a vacuum-sealed quartz tube at $1223 \mathrm{~K}$ for 15 days, and then quickly cooled to room temperature in ice water. The temperature and magnetic field dependence of macroscopic magnetism of $\mathrm{La}(\mathrm{Fe}, \mathrm{Al})_{13}$ was measured by a physical property measurement system (PPMS) of Quantum Design. The variable magnetic field and temperature field measurements of NPD with wavelengths of $2.08 \AA$ were collected in the BT- 1 neutron powder diffractometer at NIST Center for Neutron Research (NCNR). The magnetic structure refinements for all NPD data were analyzed by FULLPROF software. SARAH and BASIREPS in FULLPROF were utilized for the magnetic representational analysis.

\section{RESULTS AND DISCUSSION}

Due to the positive mixing enthalpy for the binary system of La-Fe, the composition of $\mathrm{LaFe}_{13}$ does not exist [2325]. However, due to the strong chemical affinity of $\mathrm{La}-\mathrm{Al}$, the partial substitution of $\mathrm{Al}$ for $\mathrm{Fe}$ can lower the heat of formation of $\mathrm{La}-\mathrm{Fe}$, so the cubic $\mathrm{La}\left(\mathrm{Fe}_{13-x} \mathrm{Al}_{x}\right)$ compounds can be stabilized in some regions of $x$. As previously reported, $\mathrm{La}\left(\mathrm{Fe}_{13-x} \mathrm{Al}_{x}\right)$ compounds can only be stabilized in the solution range of $1.04 \leq x \leq 7.02$, in which three magnetic states occur, i.e., AFM, ferromagnetic (FM), and micromagnetic one [26]. It has been known that the $\mathrm{La}(\mathrm{Fe}, \mathrm{Al})_{13}$-based compounds exhibit intriguing magnetic-related properties, such as magnetostriction [27], magnetovolume [28,29], magnetocaloricity [30-32], and magnetoresistance [33], due to the complex magnetism. In this study, two compositions of $\mathrm{La}\left(\mathrm{Fe}_{13-x} \mathrm{Al}_{x}\right) \quad(x=1.5$ and 1.7) at the magnetic phase boundary were prepared by an arc melting. The $\mathrm{La}\left(\mathrm{Fe}_{13-x} \mathrm{Al}_{x}\right)(x=1.5$ and 1.7) compounds maintain in a single phase with a cubic $\mathrm{NaZn}_{13}$ structure (space group: $\mathrm{Fm}-3 \mathrm{c}$ ) over the whole temperature range. There are three Wyckoff sites in $\mathrm{La}(\mathrm{Fe}, \mathrm{Al})_{13}$, i.e., $\mathrm{La}(8 \mathrm{a})(1 / 4,1 / 4,1 / 4)$, $\mathrm{Fe} 1(8 \mathrm{~b})(0,0,0)$, and $\mathrm{Fe} 2 / \mathrm{Al}(96 \mathrm{i})(0, y, z)$. As shown in Fig. 1a, one central Fe1(8b) atom and twelve surrounded $\mathrm{Fe}$ (96i) atoms form an icosahedral cluster. If taking the icosahedron as one $\mathrm{Fe}$ unit, the crystal structure of $\mathrm{La}(\mathrm{Fe}$, $\mathrm{Al}_{13}$ can be simplified to a simple CsCl-type structure [24,34].

The variable magnetic field NPD from 0 to $5 \mathrm{~T}$ at $T=$ $100 \mathrm{~K}$ was measured to study the magnetostriction property. Fig. $1 \mathrm{~b}$ shows the magnetic field $(H)$ dependence of lattice parameter $(a)$ for $\mathrm{La}\left(\mathrm{Fe}_{11.5} \mathrm{Al}_{1.5}\right)$. Intriguingly, the unit cell of $\mathrm{La}\left(\mathrm{Fe}_{11.5} \mathrm{Al}_{1.5}\right)$ expands sharply by loading magnetic field. A giant isotropic magnetostriction $(\lambda)$ of $2527 \mathrm{ppm}$ calculated by $\left(a_{5 \mathrm{~T}}-a_{0 \mathrm{~T}}\right) / a_{0 \mathrm{~T}}$ can be observed at $T=100 \mathrm{~K}$ and $H=5 \mathrm{~T}$, which is comparable to the previous macroscopic strain gauge measurement [35]. As a comparison, the magnetostriction of $\mathrm{La}\left(\mathrm{Fe}_{11.5} \mathrm{Al}_{1.5}\right)$ is even larger than that of the prototype Terfenol-D alloys of $\mathrm{Tb}_{0.3} \mathrm{Dy}_{0.7} \mathrm{Fe}_{1.9}(\lambda=1000-2000 \mathrm{ppm})[15,16]$ and comparable to that of the FeRh alloys $(\lambda=2733 \mathrm{ppm})$ [36]. To study the detailed behavior of the magnetic-field-induced lattice strain, the interatomic distances between different $\mathrm{Fe}$ atoms of icosahedron are extracted from the NPD patterns (Fig. 1c). Obviously, the nearest interatomic distance, $d_{\mathrm{Fel}-\mathrm{Fe} 2}^{1}$, has a similar tendency with the $a$, which expands with increasing magnetic field and nearly saturates at $3 \mathrm{~T}$. Therefore, the giant magnetostriction mainly comes from the magnetic-field-induced expansion of icosahedron. This result is related to the shortest Fe1-Fe2 distance, which plays a critical role in the magnetic exchange interaction [24]. Due to the expansion of icosahedron, some of the interatomic distances of $\mathrm{Fe} 2-\mathrm{Fe} 2$ increase $\left(d^{2}\right)$, or decrease $\left(d^{3}\right)$.

The temperature dependence of magnetization for $x=1.5$ and 1.7 was measured by macroscopic magnetic measurements at a magnetic field of $0.1 \mathrm{~T}$ (Fig. 2a). Obviously, $\mathrm{La}\left(\mathrm{Fe}_{11.3} \mathrm{Al}_{1.7}\right)$ shows a simple ferromagnetic behavior, but when the aluminum concentration decreases slightly to $x=1.5, \mathrm{La}\left(\mathrm{Fe}_{11.5} \mathrm{Al}_{1.5}\right)$ performs an AFM-like behavior. In this study, the composition of $\mathrm{La}\left(\mathrm{Fe}_{11.5} \mathrm{Al}_{1.5}\right)$ is chosen as the main research object. The isothermal magnetization of $\mathrm{La}\left(\mathrm{Fe}_{11.5} \mathrm{Al}_{1.5}\right)$ at selected temperatures has been measured (Fig. $2 \mathrm{~b}$ and Fig. S1). When the magnetic field is below $2 \mathrm{~T}$, the magnetization curves 

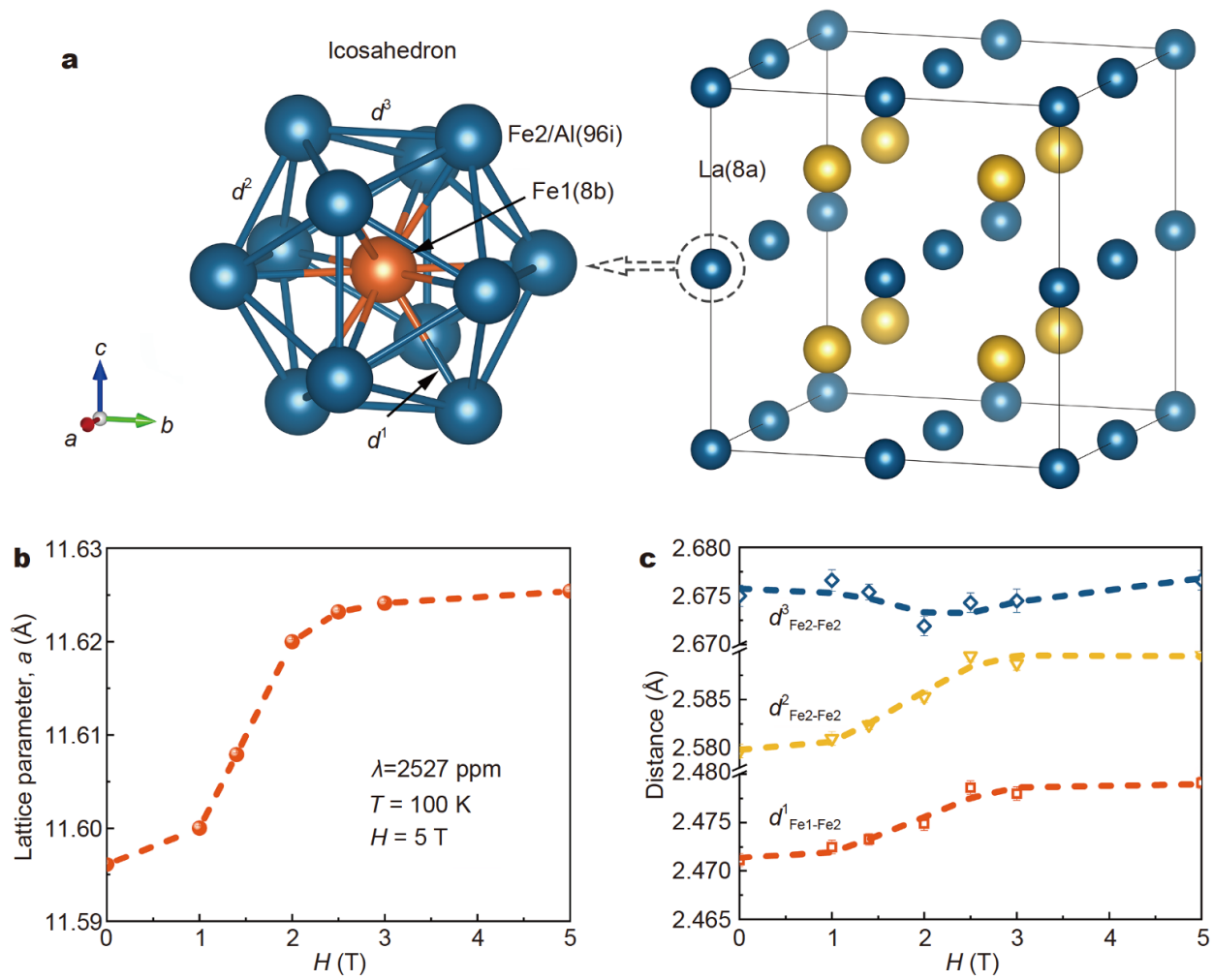

Figure 1 (a) The crystal structure of $\mathrm{La}(\mathrm{Fe}, \mathrm{Al})_{13}$ compounds. One $\mathrm{Fe}_{1}$ atom is surrounded by twelve $\mathrm{Fe}_{2} / \mathrm{Al}$ atoms, forming an icosahedron. (b) The giant magnetostriction property of $\mathrm{La}\left(\mathrm{Fe}_{11.5} \mathrm{Al}_{1.5}\right)$. The magnetic field dependence of $a$ at $T=100 \mathrm{~K}$. (c) Magnetic field dependence of Fe-Fe distances for $\mathrm{La}\left(\mathrm{Fe}_{11.5} \mathrm{Al}_{1.5}\right)$ at $T=100 \mathrm{~K}$. The $d^{1}, d^{2}$ and $d^{3}$ are marked in (a).
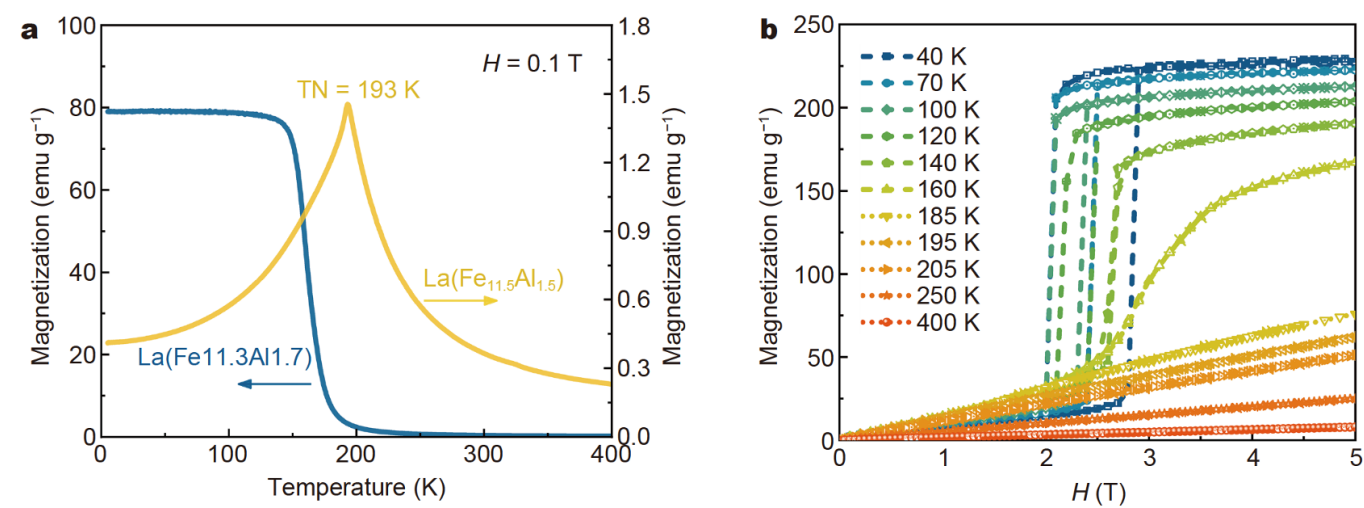

Figure 2 The macroscopic magnetism of $\mathrm{La}\left(\mathrm{Fe}_{11.5} \mathrm{Al}_{1.5}\right)$ as a function of magnetic field and temperature. (a) Temperature dependence of magnetization (FC) at $H=0.1 \mathrm{~T}$ for the $\mathrm{AFM} \mathrm{La}\left(\mathrm{Fe}_{11.5} \mathrm{Al}_{1.5}\right)$ with a comparison of the $\mathrm{FM} \mathrm{La}\left(\mathrm{Fe}_{11.3} \mathrm{Al}_{1.7}\right)$. (b) Isothermal magnetization $(M-H)$ of $\mathrm{La}\left(\mathrm{Fe} e_{11.5} \mathrm{Al} l_{1.5}\right)$ at $H=0-5 \mathrm{~T}$ and selected temperatures.

keep linear change without hysteresis, which indicates that the spin state of $\mathrm{La}\left(\mathrm{Fe}_{11.5} \mathrm{Al}_{1.5}\right)$ keeps in an AFM-like behavior at low field. However, with increasing magnetic field, a magnetic-field-induced metamagnetic transformation from the AFM-like state to the FM-like one takes place. The macroscopic magnetism of $\mathrm{La}\left(\mathrm{Fe}_{11.5} \mathrm{Al}_{1.5}\right)$ in the current magnetization curves is consistent with the previous macroscopic magnetic measurement results [37]. It should be noted that when we observe the hysteresis loop carefully, a small coercive field of $\sim 0.05 \mathrm{~T}$ can be found at $T=5 \mathrm{~K}$, which disappears gradually with increasing temperature (Fig. S2). This means that the 
magnetic state of $\mathrm{La}\left(\mathrm{Fe}_{11.5} \mathrm{Al}_{1.5}\right)$ is not a simple $\mathrm{AFM}$ structure, but a weak ferromagnetism remains.

To study the origin of giant magnetostriction in $\mathrm{La}(\mathrm{Fe}$, $\mathrm{Al}_{13}$, the variable magnetic field NPD was analyzed in detail (Fig. 3). Firstly, it needs to be noted that the magnetic structure of $\mathrm{La}(\mathrm{Fe}, \mathrm{Al})_{13}$ was ever determined to be AFM in early 1986 , which was widely used $[25,37]$. The central Fel spin parallels to the surrounded twelve $\mathrm{Fe} 2$ spins, forming one $\mathrm{Fe}$ unit. The Fe units are FM coupling within a layer, but previously assumed to be AFM coupling between the layers (Fig. 4a). As shown in Fig. 3a and Fig. S3, it is observed that some magnetic peaks increase with increasing magnetic field, while others decrease simultaneously. Such phenomenon indicates that the magnetic structure of $\mathrm{La}\left(\mathrm{Fe}_{11.5} \mathrm{Al}_{1.5}\right)$ should be composed of two components, which cannot be simply explained by the previously assumed AFM structure. Since a small ferromagnetic signal can be found in $\mathrm{La}\left(\mathrm{Fe}_{11.5} \mathrm{Al}_{1.5}\right)$, the $\mathrm{FM}$ component is added into the AFM structure to refine the NPD data. The NPD data at all magnetic fields can be fitted well by the present proposed model (Fig. 3b). As shown in Fig. $4 \mathrm{~b}, \mathrm{La}(\mathrm{Fe}, \mathrm{Al})_{13}$ exhibits a complex canting magnetic structure, which consists of two components of AFM and FM. The $M_{/ /}$and $M_{\perp}$ are used to represent the magnetic components of canting state in the parallel (the FM component) and perpendicular (the AFM component) directions, respectively.

To clearly distinguish the magnetic Bragg reflections from different components, the NPD patterns with different magnetic structures were simulated (Fig. S4). Obviously, the magnetic peaks from the FM component and AFM one are separated, which is very conducive to the analysis of magnetic structure. Due to the fact of a small ferromagnetic component, it is not easy to observe the peaks from the FM component. That is why the ferromagnetic component was often ignored at zero magnetic field in the previous studies. Intriguingly, with increasing magnetic field at $T=100 \mathrm{~K}$, the peaks of FM component are much enhanced. The coexistence of the FM and AFM component peaks proves the correctness of the present canting model. It is worth noting that both FM and AFM components exist in a single-phase canting state, rather than two separated phases, and the corresponding discussions are shown in the Supplementary information (Figs S5-S7). To the best of our knowledge, it is the first time to reveal the canting structure in $\mathrm{La}(\mathrm{Fe}, \mathrm{Al})_{13}$. The detailed magnetic structure at different fields can be refined by the NPD data. As depicted in Fig. $4 \mathrm{~b}-\mathrm{d}$, it is intriguing to observe a continuous rotation of spins by magnetic field. With increasing magnetic field, the opposite spins gradually turn to the same direction. As a result, the AFM component decreases (faint yellow), while the FM one simultaneously increases (light grey). The magnetic structure finally changes to FM structure when loading enough magnetic field.

Fig. 5a shows the magnetic field evolution of the angle $(\theta)$ of canting spins and the corresponding FM and AFM components at $100 \mathrm{~K}$. The spin moment of Fe atom almost keeps constant $\left(\left|M_{\text {Total }}\right|=\sqrt{ }\left(M_{/ /}^{2}+M_{\perp}^{2}\right)=1.47 \pm\right.$ $0.02 \mu_{B}$ ) at various magnetic fields (Fig. $5 b$ ), which means that the magnitude of total magnetic moment is not affected. However, the spin moment of canting structure is rotated by magnetic field (Fig. 5a). With increasing magnetic field, the angle $\theta$ continuously decreases. If the angle $\theta$ is close to $90^{\circ}$, it is in the AFM dominating state; however, as the angle $\theta$ is close to $0^{\circ}$, it is in the FM dominating state. Correspondingly, the $M_{/ /}$and $M_{\perp}$ change oppositely (Fig. 5a). At an intermediate field, one
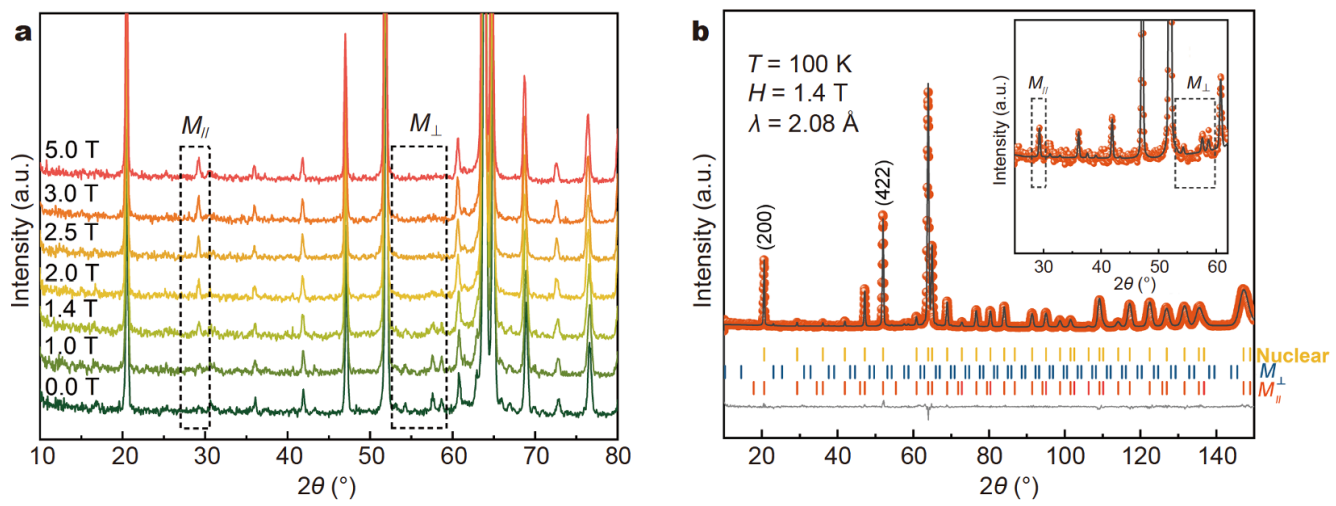

Figure 3 (a) The magnetic field dependence of NPD patterns at $T=100 \mathrm{~K}$. The dotted boxes highlight the magnetic Bragg reflections for the FM component $\left(M_{/ /}\right)$and the AFM one $\left(M_{\perp}\right)$. (b) Magnetic structure refinements of NPD patterns with a wavelength of $2.08 \AA$ for $\mathrm{La}\left(\mathrm{Fe}_{11.5} \mathrm{Al} \mathrm{l}_{1.5}\right)$ at $T=$ $100 \mathrm{~K}$ and $H=1.4 \mathrm{~T}$. The magnetic Bragg peaks are fitted by only one single-phase canting state. The inset shows the enlarged region. 


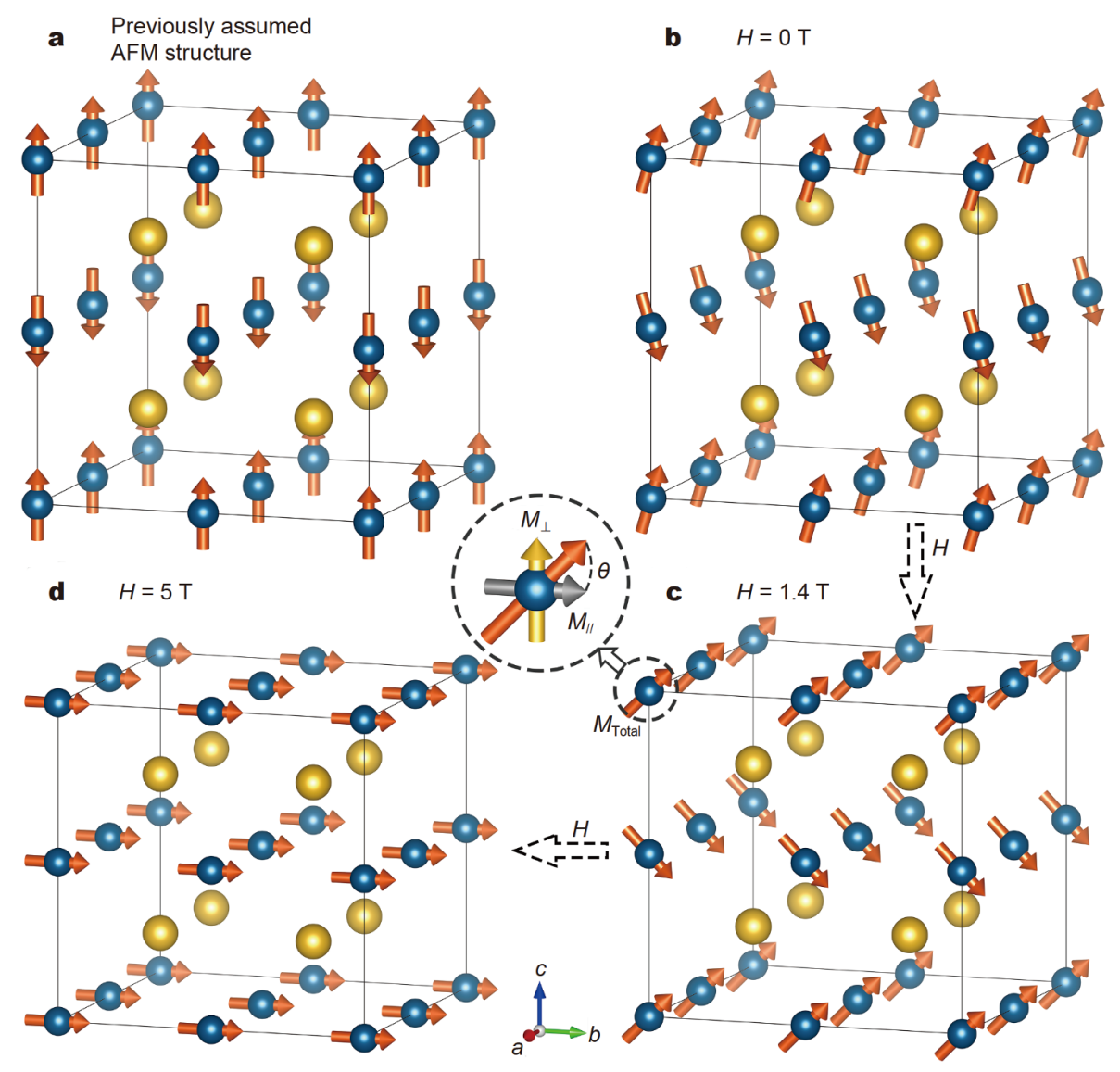

Figure 4 The spin rotation of $\mathrm{La}\left(\mathrm{Fe}_{11.5} \mathrm{Al}_{1.5}\right)$ as a function of magnetic field. (a) The previously assumed $\mathrm{AFM}$ structure in $\mathrm{La}(\mathrm{Fe}, \mathrm{Al})_{13}$. (b-d) The spin rotation in the canting structure from the AFM-dominated one to the FM one with increasing magnetic field. There are two magnetic vectors in the canting magnetic structure, i.e., the AFM $\left(M_{\perp}\right)$ and FM $\left(M_{/ /}\right)$components, which is determined by the angle of $\theta$.

can observe the coexistence of FM and AFM components such as at $H=1.4 \mathrm{~T}$ (Fig. 3a). As shown in Fig. 5c, an important fact can be observed that a strong linear correlation between $a$ and $\theta$, which directly proves that the spin rotation results in the lattice expansion. Due to the spin rotation, the FM component of spin and the lattice has a similar evolution tendency as a function of magnetic field (Fig. 1b and Fig. 5a). It suggests that the giant magnetostriction is closely related to the FM component of Fe spin, which can be evidenced by the direct spinlattice coupling between $M_{/ /}$and $a$ (Fig. $5 \mathrm{~d}$ ). The correlation can be described quantitatively by the equation of $a=a_{0}+k \cdot M_{/ /}\left(a_{0}=11.5819(18) \AA, k=0.0282(15) \AA \mu_{\mathrm{B}}^{-1}\right)$. Therefore, the origin of giant magnetostriction in $\mathrm{La}(\mathrm{Fe}$, $\mathrm{Al})_{13}$ is related to the sharp increase of FM component, which is caused by the rotation of canting magnetic spins. The nature of magnetic-field-induced rotation of magnetic spins in $\mathrm{La}(\mathrm{Fe}, \mathrm{Al})_{13}$ shows an interesting behavior similar to the role of electric-field-driven polarization rotation in the high performance piezoelectrics [38]. It is very important to reveal the common nature that the lattice can be expanded by rotation of either spin in magnetics or polarization in ferroelectrics at external field, which correspondingly triggers high performances of magnetostriction or piezoelectricity.

It is noteworthy that the origin of magnetostriction of present $\mathrm{La}(\mathrm{Fe}, \mathrm{Al})_{13}$ is different from that of $\mathrm{La}(\mathrm{Fe}, \mathrm{Si})_{13}$, which is caused by the itinerant electron metamagnetic transition, i.e., the PM-to-FM transition [27,39]. In previous studies, it is believed that the metamagnetic transformation in $\mathrm{La}(\mathrm{Fe}, \mathrm{Al})_{13}$ is the AFM-to-FM transition $[26,37]$. In fact, the previous proposed metamagnetic transformation is just the rotation of single-phase canting magnetic state, which results from the change of AFM and FM components. The spin rotation may be the essential reason for the metamagnetic transformation of most materials. For example, the AFM-to-FM transition caused by the field-induced spin reorientation has also 

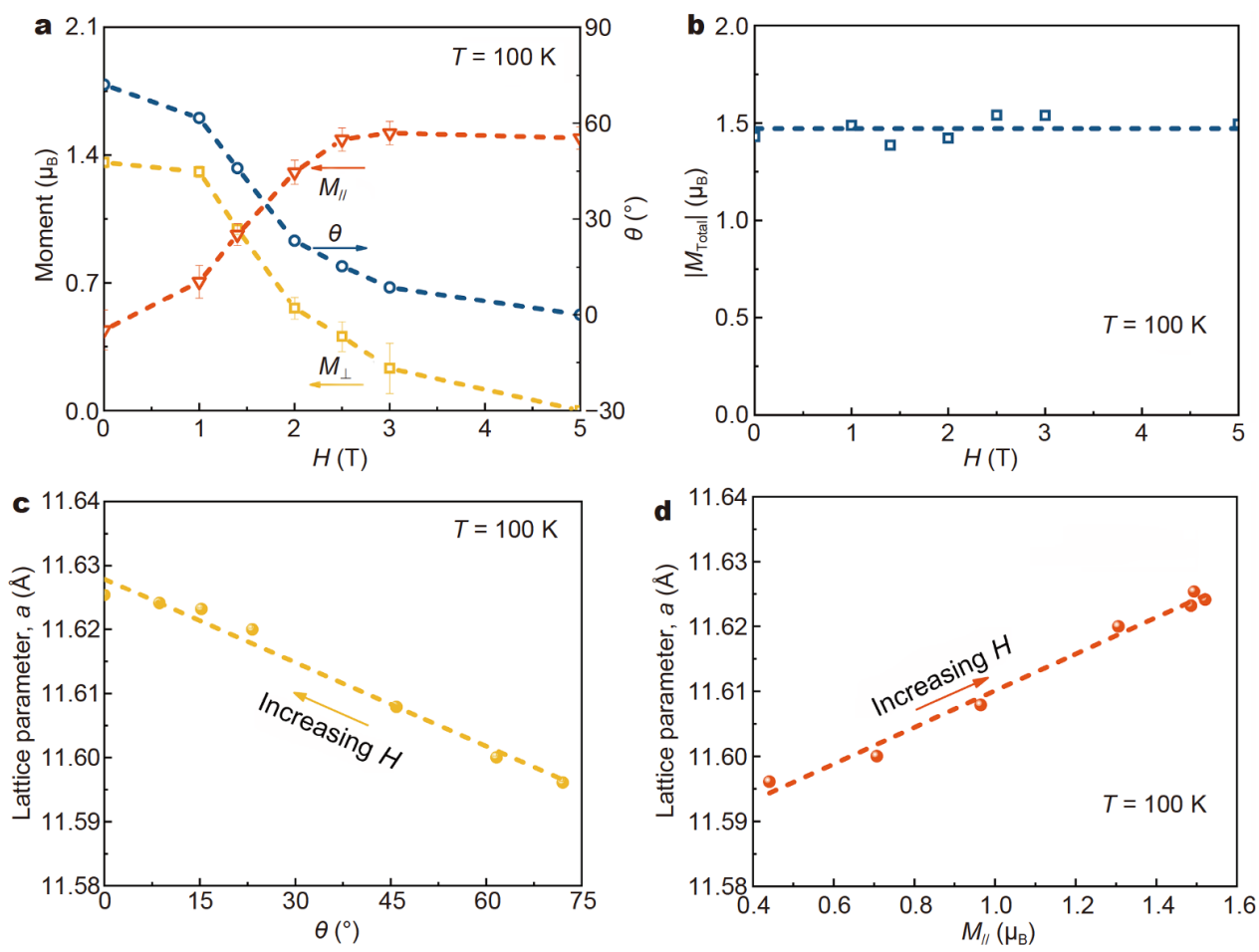

Figure 5 (a) The magnetic field evolution of $\theta$ of canting spins and the corresponding magnetic moments of FM component $\left(M_{/ /}\right)$and AFM one $\left(M_{\perp}\right)$. (b) The total moment of Fe spins at $100 \mathrm{~K}$ as a function of magnetic field. (c) A strong spin-lattice coupling between $a$ and $\theta$ of canting spins. (d) $a$ as a function of FM component moment at various magnetic fields at $100 \mathrm{~K}$.

been found in $\mathrm{EuFe}_{2} \mathrm{As}_{2}$ [40]. The present understanding in the magnetostriction mechanism is beyond the phenomenological description for magnetostriction, which will be helpful for the design of high-performance magnetostrictive materials in the future. In order to obtain high-performance magnetostrictive materials, it may be an effective pathway to obtain the unique magnetic structure which enables spin rotation smoothly driven by magnetic field.

\section{CONCLUSIONS}

In summary, the origin of giant magnetostriction $(\lambda=$ $2527 \mathrm{ppm})$ in $\mathrm{La}(\mathrm{Fe}, \mathrm{Al})_{13}$ is revealed by the direct experimental evidence for the first time. The magnetic structure of $\mathrm{La}(\mathrm{Fe}, \mathrm{Al})_{13}$ is not a simple AFM structure, but the canting antiferromagnet which performs weak ferromagnetism. The magnetic-field-triggered rotation of canting magnetic structure makes the AFM component transform to the FM one, which results in the expansion of icosahedron inside the lattice, so that the giant magnetostriction is induced. This study proves that the spin rotation may be a new strategy for obtaining large magnetostriction, which is helpful for exploring new mag- netostriction materials.

Received 17 August 2020; accepted 24 September 2020; published online 14 December 2020

1 Chopra HD, Wuttig M. Non-Joulian magnetostriction. Nature, 2015, 521: 340-343

2 Hunter D, Osborn W, Wang K, et al. Giant magnetostriction in annealed $\mathrm{Co}_{1-x} \mathrm{Fe}_{x}$ thin-films. Nat Commun, 2011, 2: 1-7

3 Yang $\mathrm{S}$, Bao $\mathrm{H}$, Zhou $\mathrm{C}$, et al. Large magnetostriction from morphotropic phase boundary in ferromagnets. Phys Rev Lett, 2010, 104: 197201

4 Koon NC, Williams CM, Das BN. Giant magnetostriction materials. J Magn Magn Mater, 1991, 100: 173-185

5 Rao WF, Xu YC, Hu CC, et al. Magnetoelastic equilibrium and super-magnetostriction in highly defected pre-transitional materials. Acta Mater, 2020, 188: 539-550

6 Náfrádi B, Keller T, Hardy F, et al. Magnetostriction and magnetostructural domains in antiferromagnetic $\mathrm{YBa}_{2} \mathrm{Cu}_{3} \mathrm{O}_{6}$. Phys Rev Lett, 2016, 116: 047001

7 Jiang S, Xie H, Shan J, et al. Exchange magnetostriction in twodimensional antiferromagnets. Nat Mater, 2020, 19: 1295-1299

8 Li MR, McCabe EE, Stephens PW, et al. Magnetostriction-polarization coupling in multiferroic $\mathrm{Mn}_{2} \mathrm{MnWO}_{6}$. Nat Commun, 2017, 8: 2037

9 Pateras A, Harder R, Manna S, et al. Room temperature giant magnetostriction in single-crystal nickel nanowires. NPG Asia 
Mater, 2019, 11: 1-7

$10 \mathrm{He} \mathrm{Y}$, Ke X, Jiang C, et al. Interaction of trace rare-earth dopants and nanoheterogeneities induces giant magnetostriction in Fe-Ga alloys. Adv Funct Mater, 2018, 28: 1800858

11 Zheng XP, Zhang PF, Li FS, et al. A study of magnetostriction, spin reorientation, and Mössbauer spectra of $\mathrm{Tb}_{0.3} \mathrm{Dy}_{0.6} \mathrm{Pr}_{0.1}\left(\mathrm{Fe}_{1-x} \mathrm{Al}_{x}\right)_{1.95}$ alloys with substitution of Fe by Al. Sci China Phys Mech Astron, 2010, 53: 449-453

12 Claeyssen F, Lhermet N, Le Letty R, et al. Actuators, transducers and motors based on giant magnetostrictive materials. J Alloys Compd, 1997, 258: 61-73

13 Quandt E, Geriach B, Seemann K. Preparation and applications of magnetostrictive thin films. J Appl Phys, 1994, 76: 7000-7002

14 Joule JP. XVII. On the effects of magnetism upon the dimensions of iron and steel bars. London Edinburgh Dublin Philos Mag J Sci, 1847, 30: 76-87

15 Clark AE, Teter JP, McMasters OD. Magnetostriction "jumps" in twinned $\mathrm{Tb}_{0.3} \mathrm{Dy}_{0.7} \mathrm{Fe}_{1.9}$. J Appl Phys, 1988, 63: 3910-3912

16 Clark AE, Belson HS. Giant room-temperature magnetostrictions in $\mathrm{TbFe}_{2}$ and $\mathrm{DyFe}_{2}$. Phys Rev B, 1972, 5: 3642-3644

17 Clark AE, Restorff JB, Wun-Fogle M, et al. Magnetostrictive properties of body-centered cubic $\mathrm{Fe}-\mathrm{Ga}$ and $\mathrm{Fe}-\mathrm{Ga}-\mathrm{Al}$ alloys. IEEE Trans Magn, 2000, 36: 3238-3240

18 He Y, Jiang C, Wu W, et al. Giant heterogeneous magnetostriction in Fe-Ga alloys: Effect of trace element doping. Acta Mater, 2016, 109: 177-186

19 Palacheva VV, Emdadi A, Emeis F, et al. Phase transitions as a tool for tailoring magnetostriction in intrinsic $\mathrm{Fe}-\mathrm{Ga}$ composites. Acta Mater, 2017, 130: 229-239

20 Chmielus M, Zhang XX, Witherspoon C, et al. Giant magneticfield-induced strains in polycrystalline Ni-Mn-Ga foams. Nat Mater, 2009, 8: 863-866

21 Sozinov A, Likhachev AA, Lanska N, et al. Giant magnetic-fieldinduced strain in NiMnGa seven-layered martensitic phase. Appl Phys Lett, 2002, 80: 1746-1748

22 Reid AH, Shen X, Maldonado P, et al. Beyond a phenomenological description of magnetostriction. Nat Commun, 2018, 9: 388

23 Tang W, Liang J, Rao G, et al. Study of AC susceptibility on the $\mathrm{LaFe}_{13-x} \mathrm{Si}_{x}$ system. Phys Stat Sol A, 1994, 141: 217-222

24 Bo LX, Altounian Z, Ryan DH. Structure and magnetic transition of $\mathrm{LaFe}_{13-x} \mathrm{Si}_{x}$ compounds. J Phys Condens Matter, 2003, 15: 73857394

25 Helmholdt RB, Palstra TTM, Nieuwenhuys GJ, et al. Magnetic properties of $\mathrm{La}\left(\mathrm{Fe}_{x} \mathrm{Al}_{1-x}\right)_{13}$ determined via neutron scattering and Mössbauer spectroscopy. Phys Rev B, 1986, 34: 169-173

26 Palstra TTM, Nieuwenhuys GJ, Mydosh JA, et al. Mictomagnetic, ferromagnetic, and antiferromagnetic transitions in $\mathrm{La}\left(\mathrm{Fe}_{x} \mathrm{Al}_{1-x}\right)_{13}$ intermetallic compounds. Phys Rev B, 1985, 31: 4622-4632

27 Fujita A, Fukamichi K. Giant volume magnetostriction due to the itinerant electron metamagnetic transition in $\mathrm{La}(\mathrm{Fe}, \mathrm{Si})_{13}$ compounds. IEEE Trans Magn, 1999, 35: 3796-3798

28 Takenaka K, Takagi H. Giant negative thermal expansion in Gedoped anti-perovskite manganese nitrides. Appl Phys Lett, 2005, 87: 261902

29 Song $\mathrm{Y}$, Sun $\mathrm{Q}, \mathrm{Xu} \mathrm{M}$, et al. Negative thermal expansion in $(\mathrm{Sc}, \mathrm{Ti}) \mathrm{Fe}_{2}$ induced by an unconventional magnetovolume effect.
Mater Horiz, 2020, 7: 275-281

30 Fujita A, Fujieda S, Hasegawa Y, et al. Itinerant-electron metamagnetic transition and large magnetocaloric effects in $\mathrm{La}\left(\mathrm{Fe}_{x} \mathrm{Si}_{1-x}\right)_{13}$ compounds and their hydrides. Phys Rev B, 2003, 67: 104416

31 Hu F, Shen B, Sun J, et al. Influence of negative lattice expansion and metamagnetic transition on magnetic entropy change in the compound $\mathrm{LaFe}_{11.4} \mathrm{Si}_{1.6}$. Appl Phys Lett, 2001, 78: 3675-3677

32 Lyubina J, Schäfer R, Martin N, et al. Novel design of $\mathrm{La}(\mathrm{Fe}, \mathrm{Si})_{13}$ alloys towards high magnetic refrigeration performance. Adv Mater, 2010, 22: 3735-3739

$33 \mathrm{Hu} \mathrm{F}$, Wang G, Wang J, et al. Magnetic entropy change and magnetoresistance in the $\mathrm{LaFe}_{11.375} \mathrm{Al}_{1.625}$ compound. J Appl Phys, 2002, 91: 7836-7838

34 Palstra TTM, Mydosh JA, Nieuwenhuys GJ, et al. Study of the critical behaviour of the magnetization and electrical resistivity in cubic $\mathrm{La}(\mathrm{Fe}, \mathrm{Si})_{13}$ compounds. J Magn Magn Mater, 1983, 36: 290296

35 Zhao Y, Huang R, Li S, et al. Giant isotropic magnetostriction in $\mathrm{NaZn}_{13}$-type $\mathrm{LaFe}_{13-x} \mathrm{Al}_{x}$ compounds. Appl Phys Lett, 2017, 110: 011906

36 Ibarra MR, Algarabel PA. Giant volume magnetostriction in the FeRh alloy. Phys Rev B, 1994, 50: 4196-4199

37 Irisawa K, Fujita A, Fukamichi K, et al. Transition between antiferromagnetic and ferromagnetic states in itinerant-electron $\mathrm{La}\left(\mathrm{Fe}_{x} \mathrm{Al}_{1-x}\right)_{13}$ compounds. Phys Rev B, 2004, 70: 214405

38 Liu H, Chen J, Fan L, et al. Critical role of monoclinic polarization rotation in high-performance perovskite piezoelectric materials. Phys Rev Lett, 2017, 119: 017601

$39 \mathrm{Xu} \mathrm{C}, \mathrm{Li} \mathrm{G}, \mathrm{Li} \mathrm{X}$, et al. Itinerant-electron metamagnetic transition and giant magnetic entropy change in $\mathrm{La}_{0.8} \mathrm{Ce}_{0.2} \mathrm{Fe}_{11.4} \mathrm{Si}_{1.6}$ compound. Chin Sci Bull, 2006, 51: 2046-2049

40 Xiao Y, Su Y, Schmidt W, et al. Field-induced spin reorientation and giant spin-lattice coupling in $\mathrm{EuFe}_{2} \mathrm{As}_{2}$. Phys Rev B, 2010, 81: 220406

Acknowledgements This work was supported by the National Natural Science Foundation of China (21825102, 22075014 and 22001014), and the Fundamental Research Funds for the Central Universities, China (06500162 and 06500145).

Author contributions Song Y and Chen J conceived this study and designed the experiments; Song Y carried out the sample preparation and other measurements; Song Y analyzed all the data of NPD with the help from other authors; Song Y and Chen J wrote the draft of the paper with the contributions from other authors; Huang Q measured and helped to analyze the NPD data; Huang R, Li L, Zhang J, Zhang S, Wang $\mathrm{S}$ and Jiang $\mathrm{Y}$ measured and analyzed the macroscopic magnetism properties; Xing X, Li L and Chen J provided useful insights on this project. All authors discussed the results and commented on the manuscript.

Conflict of interest The authors declare that they have no conflict of interest.

Supplementary information online version of the paper.
Supporting data are available in the 


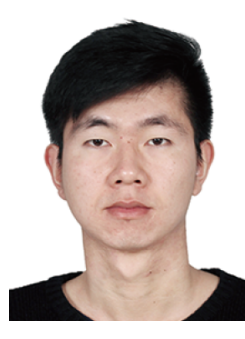

Yuzhu Song is a PhD student of the Department of Physical Chemistry at the University of Science and Technology Beijing (USTB), China. He received his $\mathrm{BSc}$ degree in metallurgical engineering from Henan University of Science and Technology in 2015. Under the supervision of Prof. Jun Chen, he has focused on the chemical design and mechanism of negative thermal expansion (NTE) in alloys. Currently, he concentrates on exploring new NTE and magnetostriction in magnetic materials, and studies their crystal/magnetic structures by means of modern methods of NPD and synchrotron radiation.

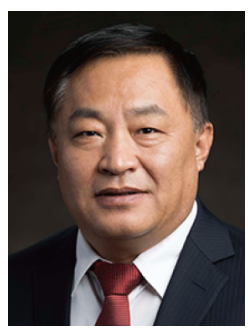

Laifeng Li received his $\mathrm{PhD}$ degree in low temperature physics from the Institute of Physics, Chinese Academy of Sciences, in 1996. In 19941995, he went to Trieste University in Italy as a visiting scholar. In 1997-1998, he joined the University of Michigan as a post-doctor. In the year of 1998, he came back to Technical Institute of Physics and Chemistry, Chinese Academy of Sciences and was promoted to a full professor. Now, he is the director of the Center of Cryogenic Materials and Technology and vice-chairman of Committee of Academic Affairs, Technical Institute of Physics and Chemistry, Chinese Academy of Sciences. His research interests are cryogenic structure and insulating materials for applied superconductors, negative thermal expansion material, thermoelectric material, as well as their mechanical and thermal properties at low temperature.

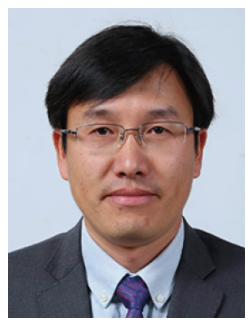

Jun Chen is currently a full professor at USTB, China. He received his BSc degree in materials science and technology from Hefei University of Technology, China, in 2001, and obtained his $\mathrm{PhD}$ degree in metallurgical physical chemistry from USTB, China, in 2007. In 2008-2009, he was financially supported by the Alexander von Humboldt Fellowship to research in the field of electrical ceramics at TU-Darmstadt, Germany. He visited the Structures Laboratory of the Tokyo Institute of Technology, Japan, as the foreign guest professor in 2015, and visited the Department of Physics and Astronomy of University of Padova, Italy as the visiting scientist in 2018. His current research interests include crystal structure, correlations with physical or chemical properties, and new materials design for ferroelectric, piezoelectric, magnetic, and negative thermal expansion solids.
自旋旋转在 $\mathrm{La}(\mathrm{Fe}, \mathrm{Al})_{13}$ 超磁致伸缩中的关键作用

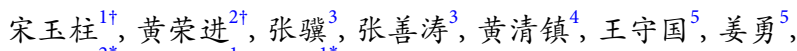
李来风 ${ }^{2 *}$, 邢献然, 陈骏 ${ }^{*}$

摘要 磁致伸缩可作为电磁能和机械能之间的高效转换途径, 是基 础研究以及技术应用等方面重要的材料性能. 然而, 在其微观成因 的理解方面仍然存在挑战, 这对磁致伸缩材料的发展非常重要. 本 文利用原位磁场和温度场下的粉末中子衍射技术首次揭示了自旋 旋转对 $\mathrm{La}(\mathrm{Fe}, \mathrm{Al})_{13}$ 超磁致伸缩的关键作用. $\mathrm{La}(\mathrm{Fe}, \mathrm{Al})_{13}$ 超磁致伸缩 性能是由磁场驱动的倾斜结构磁矩旋转引起的, 其中铁磁成分的 急剧增加促使晶格内部二十面体伸长, 进而产生巨大的磁致伸缩. 此外, 本文揭示了 $\mathrm{La}(\mathrm{Fe}, \mathrm{Al})_{13}$ 精确的倾斜磁结构特征. 本研究提供 了一种通过磁场诱导自旋旋转途径探索新型磁致伸缩功能材料的 策略. 